\title{
MORE THAN IMPOSSIBLE: NEGATIVE AND COMPLEX PROBABILITIES AND THEIR PHILOSOPHICAL INTERPRETATION
}

\author{
Vasil Penchev,vasildinev@gmail.com \\ Bulgarian Academy of Science: Institute of Philosophy and Sociology: \\ Dept. of Logical Systems and Models
}

\begin{abstract}
A historical review and philosophical look at the introduction of "negative probability" as well as "complex probability" is suggested. The generalization of "probability" is forced by mathematical models in physical or technical disciplines. Initially, they are involved only as an auxiliary tool to complement mathematical models to the completeness to corresponding operations. Rewards, they acquire ontological status, especially in quantum mechanics and its formulation as a natural information theory as "quantum information" after the experimental confirmation the phenomena of "entanglement". Philosophical interpretations appear. A generalization of them is suggested: ontologically, they correspond to a relevant generalization to the relation of a part and its whole where the whole is a subset of the part rather than vice versa. The structure of "vector space" is involved necessarily in order to differ the part "by itself" from it in relation to the whole as a projection within it. That difference is reflected in the new dimension of vector space both mathematically and conceptually. Then, "negative or complex probability" are interpreted as a quantity corresponding the generalized case where the part can be "bigger" than the whole, and it is represented only partly in general within the whole.
\end{abstract}

Key words: complex probability, Hilbert space, negative probability, probability, quantum information, vector space

\section{INTRODUCTION}

What might mean "more than impossible"? For example, that could be what happens without any cause or that physical change which occurs without any physical force (interaction) to act. Then, the quantity of the equivalent physical force, which would cause the same effect, can serve as a measure of the complex probability.

Quantum mechanics introduces those fluctuations, the physical actions of which are commensurable with the Plank constant. They happen by themselves without any cause even in principle. Those causeless changes are both instable and extremely improbable in the world perceived by our senses immediately for the physical actions in it are much, much bigger than the Plank constant.

Even more, quantum mechanics involves complex probabilities as forces explicitly as follows. Any probability distribution may be represented by its characteristic function, which is its Fourier transformation and thus a complex function sharing one and the same phase, i.e. a constant phase. If two or more probability distributions do not overlap each other, their phases are orthogonal to each other, and the common complex Hilbert space of all characteristic functions can be decomposed as a tensor product of the Hilbert spaces corresponding to each characteristic function separately. If some of them overlap each other, that decomposition is impossible. That case corresponds to a system of entangled quantum subsystems and accordingly, entangled wave functions.

The overlap of probability distributions imposes a corresponding restriction of the degrees of freedom in each space of events for the result in any of the overlapped spaces is transferred automatically in all the rest of them. That restriction of the degrees of freedom can be considered as a generalization of the physical concept of force (interaction) as to quantum 
mechanics. Indeed, any force (interaction) in the sense of classical physics causes a special kind of restriction of the degrees of freedom to a single one. Quantum force (interaction) also restricts, but to a more limited probability distribution with less dispersion and entropy rather than to a single one new value.

Particularly, that consideration interprets negative probability as a particular case of complex probability, which is what is immediately introduced.

The understanding of probability as a quantity, corresponding to the relation of part and whole, needs to be generalized to be able to include complex values. For example, probability can be thought as associable with the number of elementary permutations of two adjacent elements for a given element of a limited series to reach its last element (i.e. its upper limit) and more especially, to the ratio of that number to the corresponding number of those permutations as to the first element (i.e. the lower limit) of the series. Then, the introduction of negative probability requires only the reversion of the direction of elementary permutations from the upper limit to any element in the series.

The force can be represented in the above visualization by means of the "length" of an elementary permutation of two elements arbitrary remoted from each other. Then the length is bigger, the effective probability is bigger. The considered construction demonstrates that interpreting the probability by the well-ordering of a series, one manages to introduce complex and negative probability unambiguously as the transformation of that well-ordering at issue into others. Physical force (interaction) is then the re-ordering from one order of things into another and thus it is closely relative to complex probability.

The narrow purpose of the paper is to be introduced negative and complex probability relevant to special and general relativity and thus to events in our usual perceptive world rather than to microscopic or micro-energetic events studied by quantum mechanics (Section 3).

The prehistory and background (Section 2) include the generalization and utilization of 'negative and complex probabilities' in quantum mechanics and probability theory, and Section 4 compares their use in quantum mechanics and information, signal theory, probability theory, and special and general relativity.

\section{NEGATIVE AND COMPLEX PROBABILITY IN QUANTUM MECHANICS, SYGNAL THEORY, AND PROBABILITY THEORY}

Negative probability appears in phase-space or statistically formulated quantum mechanics; after quantum correlations, and a for wave-particle dualism.

Weyl's paper (1927) is historically first. He considered abstractly and mathematically the transformation (Weyl 1927: 116-117) reversed to the Wigner (1932: 750) function. However Weyl did not interpret the function $\mathrm{P}(\mathbf{x}, \mathbf{p})$ as probability or that it can obtain negative values.

Ville (1948) was the first who reformulated the Wigner function from the argument of a phase-space cell (space coordinates, momentum coordinates) to that of time - frequency (energy). Cohen (1995) generalized the Wigner function in a way to include Wigner's original formulation and that of Ville.

One can link to Ville and Cohen a signal modification of Wigner's phase-space reformulation of quantum mechanics and further, to Kripke's (1975) conception that a properly logical notion of truth can be introduced by infinite syntax. Feferman's "reflexive closure" (1991) will help us to clear up the syntactic "kernel" shared by two possible worlds (descriptions, theories). Wave function interpreted semantically-syntactically describes one and 
the same, but in different ways in any possible world. Thus, it represents a catalog of all possible descriptions or of all expectations about its behavior (Schrödinger 1935 (49): 823-824).

The semantic-syntactic interpretation of von Neumann's theorem (1932) about the absence of hidden parameters in quantum mechanics corresponds to the "standard" quantum logic, the base of which he founded in the same book (Neumann 1932). A semantic-syntactic interpretation of Bell's revision (Bell 1964; 1966), or in other words, defining the limits of validity for the foregoing theorem would correspond rather to "holistic semantics" (Cattaneo, Chiara, Giuntini, Paoli 2009: 193).

When the generalization of the Wigner function "was subsequently realized" (Cohen 1966: 782; 1995: 136), it was also realized that an infinite syntax can be readily generated (Cohen 1989: 943; 2008: 260).

Groenewold (1946) offered a classically statistical interpretation of quantum mechanics, however needing negative probabilities, quantizing phase space. The negative probabilities of some states do appear, but they are easily interpreted physically by the regions of partial overlap between orthogonal probabilistic distributions.

Moyal (1949) interpreted "quantum mechanics as a form of such a general statistical dynamics", in which "there is the possibility of 'diffusion' of the probability 'fluid', so that the transformation with time of the probability distribution need not be deterministic in the classical sense" (Moyal 1949: 99).

Pauli (2000: 71-72) also discussed the negative probabilities on the subject of GuptaBleurer's theory.

Einstein, Podolski, Rosen (1935) offered a "gedanken experiment", in which negative probability appears "effectively", i.e. by the restriction of the degrees of freedom (DOF) of any correlating quantum object, though not explicitly.

Neumann's theorem (1932: 167-173) about the absence of hidden parameters in quantum mechanics underlies both quantum correlation and quantum superposition as in Schrödinger "alive-and-dead cat" (Schrödinger 1935 (48): 812).

Bell's criticism (1966) about von Neumann's theorem partly rediscovered Grete Hermann's objections (1935) independently revealing the connection between causality, quantum correlation, and negative probability. Bell's inequalities (1964) imply negative probabilities, too. Kochen and Specker (1968) generalized Neumann's theorem in a link to them, utilizing a phase-space and statistical interpretation.

Negative probability for wave-particle dualism involves Einstein's papers from the "miraculous 1905" and might be discussed from the viewpoint of his "The principles of general relativity" (1918). The Kochen-Specker theorem can serve as a bridge between negative probabilities for wave-particle dualism and those for quantum correlations.

The concept of actual infinity also admits interpretation in terms of two-dimensional probability for the relativity of 'set' after Skolem (1970: 138) and the axiom of choice. That approach can be linked to Bartlett's (1944) for introducing negative probability by means of the characteristic function of random quantities and further, to Gleason's theorem (1957) about the existence of measure in Hilbert space.

Dirac (1942: 8) thought negative probability as effective rather than as real and likening it to the "negative money" in a balance. The same understanding shared Feynman in an often cited article (1991) as well as Bartlett (1944: 73). One the contrary, Mermin (1998) discussed negative probability as a kind of substance.

The brief historical review might culminate in a few philosophical questions: 
1. Whether is negative probability only a mathematical construction, or do there exist physical objects of negative probability?

2. Whether are negative probability and pure relation (such a one which cannot be reduced

to predications) equivalent, expressing one and the same case in different ways?

3. Whether does negative probability imply the physical existence of probability?

4. Can probabilities interact immediately (i.e. without any physical interaction of the things, phenomena, or events possessing those probabilities)?

5. Whether is the physical existing information deducible from the interaction of probabilities?

\section{NEGATIVE AND COMPLEX PROBABILITY LINKED TO FORCES IN SPECIAL AND GENERAL RELATIVITY}

Meaning that negative and complex probabilities which have been already linked to physical forces in quantum mechanics valid in the Plank scale, one should research that way for

them

to be introduced in special and general relativity, which should be valid in both macroscopic and microscopic (Planck) scale. This implies one to use only the kinematic formulation neglecting

the dynamical one for the latter involves mass and energy right distinguishing the scales from each other practically ${ }^{1}$ as the distances are unified as macroscopic according to the real apparatuses for quantum phenomena.

Particularly, 'force' is defined per a unit of mass (energy), and therefore equated to acceleration after kinematic consideration. 'Reference frame' is the key concept as it is properly kinematic. Still one restriction is representability in terms of quantum information, and more especially, by the concept of qubit. One needs it for the consistency and coherence of the considerations in quantum mechanics (and information) and both special and general relativity

A qubit is defined as $\alpha|0\rangle+\beta|1\rangle$, where $\alpha$ and $\beta$ are two complex numbers so that $|\alpha|^{2}+|\beta|^{2}=1$, and $|0\rangle,|1\rangle$ are two orthogonal subspaces of the complex Hilbert space (abbreviated as "cHs" further). For any two successive axes $\left(e^{i n \omega}, e^{i(n+1) \omega}\right)$ of cHs can be interpreted as those $|0\rangle,|1\rangle$, cHs or any point in it can be represented as a series of qubits $\left(Q_{n}\right)$, correspondingly "empty" or "fulfilled" by the values $\left(\alpha_{n}, \beta_{n} \in Q_{n}\right)$.

Any qubit is isomorphic to a unit ball in the usual 3D Euclidean space if two points are chosen in that ball: the one in the ball (including its surface, which is a unit sphere), and the other in the surface. That isomorphism is both elementary and crucial for our consideration for it guarantees the equivalent transfer of negative and complex probabilities between quantum mechanics (the former, "left side" of isomorphism) and both special and general relativity (the latter, "right side").

Indeed, a qubit, already as a unit ball with two points "recorded" in it, allows for another interpretation as an inertial reference frame $\left(\boldsymbol{r}_{\mathbf{0}}, \boldsymbol{v}\right)$, where the point (vector) within the ball $\left(\boldsymbol{\alpha}=\alpha_{x}, \alpha_{y}, 0\right)$ corresponds to the zero of frame $\left(\boldsymbol{r}_{\mathbf{0}}=x_{0}, y_{0}, z_{0}\right)$ and that on its surface $(\boldsymbol{\beta}=$ $\left.\beta_{x}, 0, \beta_{z}\right)$ to the speed of inertial frame $\left(\boldsymbol{v}=v_{x}, v_{y}, v_{z}\right)$. The one-to-one mapping of the components of a qubit and those of an inertial frame follows:

\footnotetext{
${ }^{1}$ Physical bodies with macroscopic mass would share the laws of quantum mechanics at almost zero values of absolute temperature, too.
} 


$$
\begin{gathered}
x_{0}=c \alpha_{x} t_{0} ; y_{0}=c \alpha_{y} t_{0} ; z_{0}=0 ; \\
v_{x}=c \beta_{x} ; v_{y}=0 ; v_{z}=c \beta_{z} .
\end{gathered}
$$

The convention " $z_{0}=0 ; v_{y}=0 "$ means that the qubit is correspondingly oriented according to the reference frame unambiguously. Any nonzero rotation of the unit ball would define a different inertial frame. The parameter " $t_{0}$ " is a conventionally chosen ordinary moment.

Further, the probability " $p$ " associable with the inertial frame may be conventionally specified as " $p=|\alpha|^{2}$ " so that $|\alpha|$ corresponds to the module of wave function just as in the Max Born interpretation of it. Indeed, then " $v=|v|=|\alpha|$ " or as "kinematic momentum" per a unit of mass (energy) might be the "length" of an elementary permutation defined as above, and its change in time would represent acceleration as "kinematic force".

Further, the unity of 'pure imaginary probability' and 'force' as above allows of still one interpretation of the relation of special and general relativity. The new interpretation is consistent to the standard one, but different from it. According to the latter, the demarcation line between special and general relativity is right the quantity of acceleration $(\boldsymbol{a})$ of the studied reference frames: zero in inertial reference frames (special relativity) and nonzero in noninertial reference (general relativity). According to the new interpretation, one should complement that distinction by the identification of any hypothetical superluminal inertial frame $\left(\boldsymbol{r}_{0}, \boldsymbol{v}:|\boldsymbol{v}|=v>c\right)$ with just one certain non-inertial subluminal reference frame $\left(\boldsymbol{r}_{\mathbf{0}}, \boldsymbol{v}, \boldsymbol{a}:|\boldsymbol{v}|=v<c\right)$. The hypothetical particles with superluminal velocity were called "tachyons". The new interpretation would add the identification of the tachyons as accelerated subluminal particles.

Indeed, special relativity identifies the pure imaginary values of speed with those of a reference frame moving with any superluminal relative velocity to an observer. The term "superluminal", which is a real value $(|\boldsymbol{v}|=v>c)$, refers to the relative speed of a hypothetical inertial reference frame to an observer in any usual, subluminal inertial reference frame. That observer should register pure imaginary values as to the velocities in the other, observed reference frame. According to the generally accepted model of special relativity in Minkowski space, time is purely imaginary unlike distance, which is real. This implies for speed to be pure imaginary as time, and acceleration to be real as distance.

Then, an observer in an inertial subluminal reference frame might not distinguish pure imaginary values of velocity from accelerations in other reference frames for both superluminal velocity and acceleration mean one and the same though expressed in two different kinds of terms: correspondingly mathematical or physical. Acceleration being a quantity different from velocity means a new dimension expressed physically. Superluminal velocity equated to pure imaginary velocity according to special relativity means a new dimension expressed mathematically, namely that of imaginary axis in relation to the real one.

Further, the accelerated inertial frames already according to general relativity implies a force field indistinguishable from the gravitational one in turn represented by curving Minkowski space to pseudo-Riemannian one. Any tensor associable with a point in pseudoRiemannian space is representable as the tensor product of vectors of Minkowski space as in its real as in its imaginary domain. Particularly, the tensor of curvature in a point transforms a vector to another between the imaginary and real domain.

Then, the formalism of general relativity is interpretable as presenting transformations within both domains of Minkowski space separately or together unlike that of special relativity restricted only to the one: that associated to the subluminal area usually identified with the 
imaginary cone (domain). The pair of contra- and covariant 4-vectors as to pseudo-Riemannian space is isomorphic to that of4-vectors in each of the real and imaginary domain as to Minkowski space.

\section{THE PHILOSOPHICAL INTERPRETATION: THE UNITED NATURE OF FORCES AND PROBABILITIES IN QUANTUM MECHANICS AND INFORMATION, SPECIAL AND GENERAL RELATIVITY, AND PROBABILITY AND INFORMATION THEORY}

The introduction of complex probabilities unifies forces and probabilities as two dimensions, whether mathematically or physically interpreted, of one and the same nature, that of complex probabilities. Then both "more than impossible" and even the "squire root of that more than impossible" acquire a clear mathematical and physical meaning:

All physical forces (interactions) as in quantum mechanics (and therefore in the Standard model) and in special or general relativity are a particular case of the generalized probabilities and relative to the classical, one-dimensional probabilities. Furthermore, the two dimensional (or complex) probabilities unify the subjective probabilities of the observer and the objective probabilities of the observed, and even the one dimension might be ascribed to the former, the other to the latter. This allows of unifying further the concept of 'observer' in relativity and quantum mechanics.

In the final analysis, the border between the physical theories of quantum mechanics and those of special and general relativity melts in probability and information theory turning out to underlie both.

\section{REFERENCES:}

Bartlett, M. (1945) "Negative Probability," Mathematical Proceedings of the Cambridge Philosophical Society 41 (1): 71-73.

Bell, J. (1964) "On the Einstein - Podolsky - Rosen paradox," Physics (New York) 1 (3): 195-200.

Bell, J. (1966) "On the Problem of Hidden Variables in Quantum Mechanics," Reviews of Modern Physics 38 (3): 447-452.

Cattaneo, G., M. Chiara, R. Giuntini, F. Paoli (2009) "Quantum Logic and Nonclassical Logics," in Handbook of Quantum Logic and Quantum Structures. Quantum Logic (eds. K. Engesser, D. Gabbay, D. Lenmann). Amsterdam, etc.: Elsevier, pp. 127-226.

Cohen, L. (1966) "Generalized phase-space distribution functions," Journal of Mathematical Physics 7 (5): 781-786.

Cohen, L. (1989) "Time-Frequency Distributions - A Review," Proceedings of the IEEE 77 (7): 941-981.

Cohen, L. (1995) Time-Frequency Analysis. New York: Prentice-Hall

Cohen, L. (2008) The Weyl transformation and its generalization. Rendiconti del Seminario Matematico. Università Politecnico di Torino 66 (4): 259-270.

Dirac, P. (1942) Bakerian Lecture. The Physical Interpretation of Quantum Mechanics. Proceedings of the Royal Society of London A 180 (980): 1-40.

Einstein, A. (1905) "Ist die Trägheit eines Körpers von seinem Energieinhalt abhängig?" Annalen der Physik 18 (13): 639-641.

Einstein, A. (1905) "Über einen die Erzeugung und Verwandlung des Lichtes betreffenden heuristischen Gesichtspunkt," Annalen der Physik 17 (6): 132-148.

Einstein, A. (1918) "Prinziplelles zur allgemeinen Relativitätstheorie," Annalen der Physik 55 (4): 241-244.

Einstein, A., B. Podolsky and N. Rosen (1935) "Can Quantum-Mechanical Description of Physical Reality Be Considered Complete?” Physical Review 47 (10): 777-780. 

$1-49$.

Feferman, S. (1991) "Reflecting and Incompleteness," The Journal of Symbolic Logic 56 (1):

Feynman, R. (1991) "Negative Probability," in Quantum Implications: Essays in Honour of David Bohm (eds. B.Hiley, D. Bohm, D. Peat). London - New York, Routledge, pp. 235-248.

Gleason, A. (1957) "Measures on the Closed Subspaces of a Hilbert Space," Journal of Mathematics and Mechanics 6 (6): 885-893.

Groenewold, H. (1946) "On the Principles of Elementary Quantum Mechanics," Physica 12 (7): 405-460.

Hermann, G. (1935) "The circularity in von Neumann's proof," (Translation by Michiel Seevinck of "Der Zirkel in NEUMANNs Beweis", section 7 from the essay by Grete Hermann, "Die Naturphilosophischen Grundlagen de Quantenmechanik,"Abhandlungen der fries'schen Schule 6, 1935) - http://www.phys.uu.nl/igg/seevinck/trans.pdf

Kochen, S., E. Specker. (1968) "The problem of hidden variables in quantum mechanics," Journal of Mathematics and Mechanics 17 (1): 59-87. 716.

Kripke, S. (1975) "Outline of a Theory of Truth," The Journal of Philosophy 72 (19), 690-

Mermin, D. (1998) “What Is Quantum Mechanics Trying to Tell Us?" American Journal of Physics 66 (9): 753-767.

Moyal, J. (1949) Quantum mechanics as a statistical theory. Proceedings of the Cambridge Philosophical Society 45 (1): 99-124.

Neumann, J. von. (1932) Mathematische Grundlagen der Quantenmechanik. Berlin: Springer.

Pauli, W. (2000). Pauli lectures on physics. Vol. 6. Selected topics in field quantization. (ed. C. Enz.), New York, Courier Dover Publications, pp. 1-73.

Schrödinger, E. (1935) Die gegenwärtige situation in der Quantenmechanik. Die Naturwissenschaften 23 (48): 807-812; 23 (49): 823-828; 23 (50): 844-849.

Skolem, T. (1970) "Einige Bemerkungen zur axiomatischen Begründung der Mengenlehre." in: Selected works in logic (ed. E. Fenstad), Oslo, etc., Univforlaget, pp. 137-152.

Ville, J. (1948) "Théorie et Applications de la Notion de Signal Analytique," Cables et Transmission 2A (janvier): 61-74.

Weyl, H (1927) "Quantenmechanik und Gruppentheorie," Zeitschrift für Physik 46 (1-2): 1-46.

Wigner, E. (1932.) "On the Quantum Correction for Thermodynamic Equilibrium," Physical Review 40 (5): 749-759. 